\title{
The Traveling Salesman Problem with Precedence Constraints and Binary Costs
}

\author{
L. Bianco ${ }^{\ddagger}, P$. Dell'Olmo ${ }^{\ddagger}, S$. Giordani\$ \\ $\ddagger$ Electronic Eng. Dept., § "V. Volterra" C.-Univ. of Rome "Tor Vergata" \\ Viale della Ricerca Scientifica, I-00133 Rome, Italy. Ph.: +39-6-77161. \\ Fax: +39-6-7716461. email: giordani@iasi.rm.cnr.it
}

\begin{abstract}
Given a set of $n$ elements $V=\{1,2, \ldots, n\}$ and a precedence relation $<_{P} \subseteq V \times V$ defined on $V$, where $i<_{P} j$ or $(i, j) \in<_{P}$ means that $i$ precedes $j$, let us consider the complete digraph $G=(V, A)$ with no loops and with $(j, i) \notin A$ if and only if $i<_{P} j$. Let be assigned a cost $c_{i j} \in\{0,1\}$ to each $\operatorname{arc}(i, j) \in A$ with $c_{i j}=0$ if and only if $(j, i) \notin A$. Let $G^{\prime}$ be the digraph obtained from $G$ adding a dummy vertex $s$, the Traveling Salesman Problem with Precedence Constraints and Binary Costs (TSP-PC-BC) is to find a hamiltonian tour of minimum cost in $G^{\prime}$, starting from vertex $s$, visiting each vertex $i \in V$ after having visited every vertex $j \in V$ that must precede $i$ and returning to vertex $s$. In this paper we investigate the TSP-PC-BC using a different approach from those used in previous works on TSP: we replace the graph $G^{\prime}$ with a partially ordered set $P=\left(V,<_{P}\right)$, defined by $<_{P}$ on $V$ and reformulate the problem as the Jump Number Problem of $P$. We also propose exact and heuristic solution algorithms. Computational results on a set of randomly generated test problem are discussed.
\end{abstract}

Keywords

TSP, binary costs, posets, linear extensions, heuristic, dynamic programming.

\section{INTRODUCTION}

Given a set of $n$ elements $V=\{1,2, \ldots, n\}$ and a precedence relation $<_{P} \subseteq V \times V$ defined on $V$, where $i<_{P} j$ or $(i, j) \in<_{P}$ means that $i$ precedes $j$, let us consider the complete digraph $G=(V, A)$ with no loops and with $(j, i) \notin A$ if and only if $i<_{P} j$. Let be assigned a cost $c_{i j} \in\{0,1\}$ to each arc $(i, j) \in A$ with $c_{i j}=0$ if and only if $(j, i) \notin A$. Let $G^{\prime}$ be the digraph obtained from $G$ adding a dummy vertex $s$, the Traveling Salesman Problem with Precedence Constraints and Binary Costs (TSP-PC-BC) is to find a hamiltonian tour of minimum cost in $G^{\prime}$, starting from vertex $s$, visiting each vertex $i \in V$ after having visited every vertex $j \in V$ that must precede $i$ and returning to vertex $s$.

The Traveling Salesman Problem with Precedence Constraints is known to be $\mathcal{N} \mathcal{P}_{\text {- }}$ 
hard and has several practical applications in sequencing and routing problems, like crew scheduling and vehicle routing. It has been investigated by Bianco Mingozzi and Ricciardelli (1994) and Savelsberg (1990) for the general case and in the dial-a-ride problem version by Psaraftis $(1980,1983)$. At the best of our knowledge the particular case TSPPC-BC has not yet been studied, and questions about this problem, e.g. computational complexity, are not yet explicitly answered.

In this paper we investigate the TSP-PC-BC using a different approach from those used in previous works: we replace the graph with a partially ordered set $P=\left(V,<_{P}\right)$, defined by the precedence relations between the elements of $V$ and reformulate the problem as the jump number problem of $P$ as explained in the sequel.

Given the precedence relation $<_{P}$, let us consider the relational set $\left(V,<_{P}\right)$, that is a partially ordered set (poset) $P=\left(V,<_{P}\right)$, since the relation $<_{P}$ is irreflexive, antisymmetric and transitive. Notationally, $u<_{P} v$ means that $u$ precedes $v$, while $u \|_{P} v$ means that $u$ and $v$ are incomparable and $u \not_{P} v$ simply means that $u$ does not precede $v$, regardless if $v$ precedes or not $u$; moreover, two elements $u, v \in V$ are comparable $\left(u \sim_{P} v\right)$ if $u<_{P} v$ or $v<_{P} u$. Given a partially ordered set $P=\left(V,<_{P}\right)$, a linear extension $L=\left(V,<_{L}\right)$ of $P$ is a total order on the same ground set $V$ of $P$, such that each couple of elements $u, v \in V$ for which $u<_{P} v$ implies $u<_{L} v$. Given two consecutive elements $u, v \in L$ the couple $(u, v)$ is a jump or setup of $L$ if $u, v$ are not comparable in $P$; the jump number of $L$, denoted by $s(L, P)$ is the number of jumps in $L$, while the jump number of $P$, denoted by $s(P)$, is the minimum of $s(L, P)$ taken over all linear extensions of $P$. A linear extension $L$ of $P$ is called jump optimal if $s(L, P)=s(P)$. The jump number problem of $P$ consists in determining $s(P)$ and a jump optimal linear extension $L$ of $P$.

For the above formulation some complexity results are known. In fact, the jump number problem is known to be $\mathcal{N} \mathcal{P}$-hard for general posets (Pulleyblank, 1981), even if there are some classes of posets for which the problem may be optimally solved in polynomial time. In the non polynomial cases, an exact algorithm for general posets has been developed by Bianco, Dell'Olmo and Giordani (1994a) and approximation algorithms exist only for interval orders (Mitas, 1991).

In this work, we show that the TSP-PC-BC is equivalent to the Jump Number problem of $P=\left(V,<_{P}\right)$, proving that TSP-PC-BC remains $\mathcal{N P}$-hard. We propose both heuristic and exact algorithms, which solve TSP-PC-BC finding a jump optimal linear extension of $P$. Computational results show that the algorithms presented in this paper overcome the performances of the algorithm proposed by Bianco, Dell'Olmo and Giordani (1994a).

\section{THE TSP-PC-BC AND THE JUMP NUMBER PROBLEM}

Let $G=(V, A)$ be a complete digraph (i.e. a directed graph with at least one arc joining every pair of vertices) without loops and with the following cost assignment:
(a)
(b)
$c_{i j} \in\{0,1\}$
(c)
$c_{i j}=0$ if and only if $(i, j) \in A$ and $(j, i) \notin A$;
$c_{i k}+c_{k j} \geq c_{i j}$.

Furthermore, let $<_{P}$ be a binary relation on the elements of $V$ defined as:

$i<_{P} j$ for $c_{i j}=0$. 
Proposition 1 The relation $<_{P}$ stated as above on the graph $G=(V, A)$ defines a poset $P=\left(V,<_{P}\right)$ if and only if the costs of the arcs of $G$ fulfill properties $(a),(b)$ and (c).

Proof. Let $G_{P}$ be the digraph obtained from $G$ considering only the zero cost arcs. The binary relation $<_{P}$ defined on the set $V$ by the arc set of $G_{P}$ is transitive by property (c), antisymmetric by property (b) and irreflexive since $G_{P}$ is with no loops. Therefore $P=\left(V,<_{P}\right)$ is a poset and, furthermore, $G_{P}$ is a transitive orientation of the edges of a comparability graph (Möring, 1985).

Vice-versa, given a poset $P=\left(V,<_{P}\right)$, let denote with $G^{\prime \prime}=\left(V, A^{\prime \prime}\right)$ the complete symmetric digraph with the following arc costs: $c_{i j}=0$ and $c_{j i}=\infty$ for $i<_{P} j ; c_{i j}=1$ and $c_{j i}=1$ for $i \|_{P} j ; c_{i i}=\infty$. Removing from $G^{\prime \prime}$ the infinity cost arcs we obtain a complete digraph $G=(V, A)$ without loops. By definition the costs of the arcs in $A$ respect properties (a) and (b). Moreover, from the transitive property of the binary relation $<_{P}$ the property (c) follows.

Then, we may say that the poset $P=\left(V,<_{P}\right)$ and the complete digraph $G=(V, A)$ defined above with the relation $<_{P}$ as in (1), are equivalent. See, for example, the digraph $G=(V, A)$ and the equivalent poset $P=\left(V,<_{P}\right)$ depicted in Figure 1a. Moreover, it is easily proved that:

Corollary 1 Let be given $P=\left(V,<_{P}\right)$ and the equivalent representation $G=(V, A)$. It results $i \|_{P} j$ if and only if $c_{i j}=c_{j i}=1$ in $G$.

Corollary 2 Let us consider $P=\left(V,<_{P}\right)$ and the equivalent representation $G=(V, A)$. It results $i \not_{P} j$ if and only if $(j, i) \in A$.

Proposition 2 Given $G=(V, A)$ and the equivalent poset $P=\left(V,<_{P}\right)$, a total order $\Pi=\left(a_{1}, a_{2}, \ldots, a_{n}\right)$ of the elements of $V$ is a linear extension $L$ of $P$ if and only if $\Pi$ corresponds to a hamiltonian path $H_{L}$ in $G$ respecting the precedence relation $<_{P}$.

Proof. Let us consider a total order $\Pi$ of the elements of $V$ and suppose that it represents a linear extension $L=a_{1}, a_{2}, \ldots, a_{n}$ of $P . L$ defines a path in $G$ since for each couple of consecutive elements $\left(a_{i}, a_{i+1}\right)$ in $L$ it results $a_{i}<_{P} a_{i+1}$ or $a_{i} \|_{P} a_{i+1}$, that is $\left(a_{i}, a_{i+1}\right) \in A$ from Corollary 1. Actually, $H_{L}=\left(a_{1}, a_{2}, \ldots, a_{n}\right)$ is a hamiltonian path of $G$ since $L$ contains every element of $P$ exactly once. Moreover, by definition of linear extension, $H_{L}$ respects the precedence relations defined by $P$.

Vice-versa, let us consider a hamiltonian path $H_{L}=\left(a_{1}, a_{2}, \ldots, a_{n}\right)$ in $G=(V, A)$. It represents a total order of the elements of $V$; to represent a linear extension $L=$ $a_{1}, a_{2}, \ldots, a_{n}$ of $P$ it is necessary that for each couple $\left(a_{i}, a_{i+1}\right)$ of $H_{L} a_{i+1} \not_{P} a_{i} ;$ but this is the case, since each arc $\left(a_{i}, a_{i+1}\right) \in A$ implies that $a_{i+1} \nless_{P} a_{i}$, by Corollary 2. Moreover, $H_{L}$ has to respect the precedence relation on the elements of $V$, otherwise in $H_{L}=\left(a_{1}, \ldots, a_{i}, \ldots, a_{i+k}, \ldots, a_{n}\right)$ there will be at least a couple of elements (say $a_{i}$ and $a_{i+k}$ ) for which $a_{i+k}<_{P} a_{i}$, that is $L$ is not a linear extension.

Corollary 3 The jump number $s(L, P)$ of a linear extension $L$ of a poset $P=\left(V,<_{P}\right)$ is equal to the cost of the corresponding hamiltonian path $H_{L}$ in the equivalent $G=(V, A)$. 


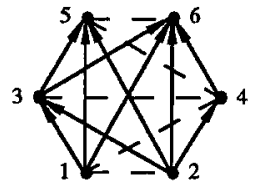

$\mathrm{G}=(\mathrm{V}, \mathrm{A})$

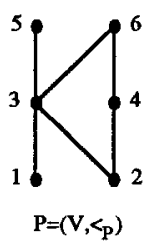

$\longrightarrow \quad$ : arc with cost $0 ;<_{\mathrm{p}}$

- $\quad$ : symmetric ares with cost 1

(a)
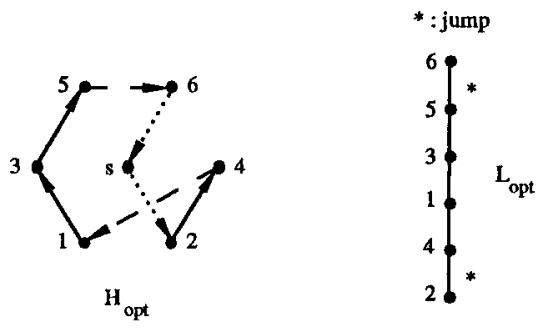

(b)

Figure 1 (a): A digraph $G=(V, A)$ with relation $<_{P}$ and the Hasse diagram of the equivalent poset $P ;(\mathrm{b})$ : an optimal solution $H_{\text {opt }}$ for the TSP-PC-BC on $G^{\prime}=(V U$ $\{s\}, A \cup B)$ and an optimal solution $L_{\text {opt }}$ for the jump number problem on $P$.

Proof. It follows directly from the costs of the arcs in $A$. In fact, by Corollary 1 the arcs in $H_{L}$ whose cost is not equal to zero but one are only those arcs corresponding to incomparable couples of elements in $P$, that the jumps in $L$. Therefore the cost of $H_{L}$ is equal to the jump number of $L$.

Now, we may assert that finding the jump number of a poset $P$ is equivalent to find a minimum cost hamiltonian path, respecting the precedence relations defined by $P$, in a complete digraph $G=(V, A)$ without loops and binary costs for the arcs. Moreover, let $G^{\prime}=(V \cup\{s\}, A \cup B)$ be the digraph obtained adding to $G$ a dummy vertex $s$ and the zero cost arcs in $B=\bigcup_{v \in V}\{(s, v),(v, s)\}$. The jump number problem on $P=\left(V,<_{P}\right)$ is equivalent to the Traveling Salesman Problem with Precedence Constraints and Binary Costs (TSP-PC-BC) on $G^{\prime}$, that is to find a minimum cost hamiltonian tour starting from vertex $s$, visiting every vertex $i$ for which $i<_{P} j$ before $j$ and returning to $s$. An example is given in Figure 1.

Theorem 1 The TSP-PC-BC is $\mathcal{N P}$-hard.

Proof. It follows from the fact that the jump number problem, which is $\mathcal{N} \mathcal{P}$-hard (Pulleyblank, 1981), polynomially transforms to TSP-PC-BC as explained before. 


\section{HEURISTIC ALGORITHM}

First of all, we will report some further notations and definitions. Given a poset $P=$ $\left(V<_{P}\right)$, a subposet $P \mid X$ of $P$ is a subset of $P$ with the induced order. A chain $C$ is a set of pairwise comparable elements of $P$, that is a subposet of $P$ which is a total order of $P$; moreover, let $\operatorname{Min}(P)$ and $\operatorname{Max}(P)$ be the sets of minimal and maximal elements of $P$, respectively. Given an element $v \in P$, let us consider the sets $\operatorname{Pred}(v)$ and $S u c(v)$ : we say that $v$ is down-accessible (up-accessible) if $\operatorname{Pred}(v)(S u c(v))$ is a chain in $P$. Furthermore, a chain $C$ in $P$ will be called greedy $\left({ }^{*}\right.$ greedy) if the top element of the chain $C$, denoted with $\sup _{P}(C)$ (the bottom element of the chain $C$, denoted with in $f_{P}(C)$ ) is a maximal down-accessible (up-accessible) element of $P$. We will consider greedy chains $C_{u_{k}}$ for which $u \in C_{u_{k}}$ and $u \in \operatorname{Min}(P)$, and *greedy chains $C_{v_{l}}^{*}$ for which $v \in C_{v_{l}}^{*}$ and $v \in \operatorname{Max}(P)$, and we will call them, respectively, greedy starting chains and * greedy ending chains of $P$. Moreover, let us say that $v \in P$ is $S$-maximal if $\forall u \in P, S u c(u) \subseteq S u c(v)$. It is clear that each S-maximal element of $P$ is also a member of $\operatorname{Min}(P)$. Likewise, let us define $v \in P$ an $S^{*}$-maximal element of $P$ if $\forall u \in P, \operatorname{Pred}(u) \subseteq \operatorname{Pred}(v)$ (obviously $v \in \operatorname{Max}(P)$ too).

It is known (see Faigle and Schrader 1985) that given a poset $P$, if the set $S_{\max }(P)$ of Smaximal elements of $P$ exists then there also exists a jump optimal linear extension whose starting subset is composed by $S_{\max }(P)$, regardless the order in which the S-maximal elements are chosen. A similar result may be obtained considering the $S^{*}$-maximal elements of $P$ and choosing them as the ending subset of a linear extension. Consider the following situation. Given a poset $P=\left(V,<_{P}\right)$, suppose that:

the S-maximal $S_{\max }(P)$ and $\mathrm{S}^{*}$-maximal $S_{\max }^{*}(P)$ sets of $P$ exist and do not intersect, and, considering the poset $P_{1}=P \mid\left(V-\left(S_{\max }(P) \cup S_{\max }^{*}(P)\right)\right)$, suppose that:

the S-maximal $S_{\max }\left(P_{1}\right)$ and $\mathrm{S}^{*}$-maximal $S_{\max }^{*}\left(P_{1}\right)$ sets of $P_{1}$ exist and do not intersect. (ii)

Proposition 3 If hypotheses (i) and (ii) hold, there exists a jump optimal linear extension $L=L_{0}^{s} \oplus L_{1} \oplus L_{0}^{e}$ of $P$, where $L_{0}^{s}$ is any linear order of $S_{\max }(P)$ and $L_{0}^{e}$ is any linear order of $S_{\max }^{*}(P)$, and $L_{1}$ is a jump optimal linear extension of $P_{1}$, whose starting subset and ending subset are, respectively, a particular linear order of $S_{\max }\left(P_{1}\right)$ where the starting element is $u_{1} \in S_{\max }\left(P_{1}\right)$, such that $\sup _{L}\left(L_{0}^{s}\right)<_{P} u_{1}$ if such $u_{1}$ exists, and a particular linear order of $S_{\max }^{*}\left(P_{1}\right)$, where the ending element is $z_{1} \in S_{\max }^{*}\left(P_{1}\right)$, such that $z_{1}<_{P}$ in $f_{L}\left(L_{0}^{e}\right)$ if such $z_{1}$ exists.

In the cases in which the set $S_{\text {max }}\left(P_{1}\right)\left(S_{\text {max }}^{*}\left(P_{1}\right)\right)$ is empty or there is no elements $u_{1} \in$ $S_{\max }\left(P_{1}\right)$ such that $\sup _{L}\left(L_{0}^{s}\right)<_{P} u_{1}\left(z_{1} \in S_{m a x}^{*}\left(P_{1}\right)\right.$ such that $z_{1}<_{p}$ inf $\left.f_{L}\left(L_{0}^{e}\right)\right)$, it is possible to consider the set of greedy starting chains $\left\{C_{u_{k}}\right\}$ of $P_{1}$ for each $u \in \operatorname{Min}\left(P_{1}\right)$ (the set of * greedy ending chains $\left\{C_{v_{l}}^{*}\right\}$ of $P_{1}$ for each $v \in \operatorname{Max}\left(P_{1}\right)$ ); from this set of chains it will be convenient to choose the greedy starting chain $C_{q}$ of maximum size such that $\sup _{L}\left(L_{0}^{s}\right)<_{P}$ in $f_{P}\left(C_{q}\right)$, if it exists (the ${ }^{*}$ greedy ending chain $C_{r}^{*}$ of maximum size such that $\sup _{P}\left(C_{r}^{*}\right)<_{P}$ in $f_{L}\left(L_{0}^{e}\right)$, if it exists) and such that $C_{q} \cap C_{r}^{*}=\emptyset$.

All these considerations lead to the definition of a heuristic algorithm, in which a linear extension of $P$ is constructed from the bottom and from the top, adding at each iteration two disjointed subsets of the elements of $P$, chosen, respectively, from the S-maximal set and the greedy starting chains, and from the $\mathrm{S}^{*}$-maximal set and the ${ }^{*}$ greedy ending chains of the residual poset. For a complete description of the proposed heuristic algorithm see (Bianco, Dell'Olmo and Giordani, 1994b). 


\section{EXACT ALGORITHM}

In the following, we design an exact algorithm based on dynamic programming. To this end, we illustrate some further properties of a poset.

Proposition 4 Given a poset $P=\left(V,<_{P}\right)$, for any subposet $P \mid X$, where $X$ is a subset of the ground set $V$ of $P$, the jump number of $P \mid X$ is $s(P \mid X) \leq s(P)$.

Theorem 2 Given a poset $P=\left(V,<_{P}\right)$ for each greedy starting chain $C$ of $P$ it results $s(P \mid(V-C)) \leq s(P) \leq s(P \mid(V-C))+1$.

Corollary 4 The minimum jump number in some linear extension $L$ of $P$ with $C$ as starting subset is equal to $s(P \mid(V-C))+1$.

Theorem 3 Given a poset $P=\left(V,<_{P}\right)$ there exists a jump optimal linear extension $L$ of $P$, whose starting subset is composed by a greedy starting chain $C$.

On the basis of Theorem 3, a jump optimal linear extension may be found generating only linear extensions which have a greedy starting chain as a starting subset. Let us consider the couple $\left(X, C_{i}\right)$ representing the subposet $P \mid X$ and an its greedy starting chain $C_{i}$. Let $f\left(X, C_{i}\right)$ be the minimum jump number in some linear extensions of $P \mid X$ with $C_{i}$ as starting subset. Let $\mathcal{G}=(\mathcal{S}, \mathcal{A})$ be a digraph (called state space graph) whose vertex set $\mathcal{S}$ contains the states $\left(X, C_{i}\right)$, and whose arc set $\mathcal{A}=\left\{\left(\left(X, C_{i}\right),\left(X^{\prime}, C_{j}\right)\right): X=X^{\prime} \cup C_{i}\right\}$ represents all feasible transitions from one state $\left(X, C_{i}\right) \in \mathcal{S}$ to another $\left(X^{\prime}, C_{j}\right) \in \mathcal{S}$. The cost of each arc $\left(\left(X, C_{i}\right),\left(X^{\prime}, C_{j}\right)\right) \in \mathcal{A}$ is equal to 1 , as for Corollary 4 . Then, the dynamic programming recursion that defines $f\left(X, C_{i}\right)$ may be stated as follows:

$f\left(X, C_{i}\right)=\min _{\left\{\left(X^{\prime}, C_{j}\right) \in \mathcal{S}:\left(\left(X, C_{i}\right),\left(X^{\prime}, C_{j}\right)\right) \in \mathcal{A}\right\}}\left\{f\left(X^{\prime}, C_{j}\right)+1\right\}$,

initialized by $f(Y, Y)=0$, since $P \mid Y$ is a chain. The optimal solution to the problem is then given by $z^{*}=\min _{\left(V, C_{v}\right) \in \mathcal{S}} f\left(V, C_{v}\right)$.

Moreover, let us consider the following two dominance rules. We refer to a poset $P$ that, without loss of generality, is not a chain. The dominance rules may be respectively established on the basis of the following lemmas, which may be easily proved:

Lemma 1 Given a poset $P=\left(V,<_{P}\right)$ and a greedy starting chain $C$ of $P$, consider the element $a=\sup _{P}(C)$. If $a \in M a x(P)$ then $s(P)=s(P \mid(V-C))+1$, that is there exist an optimal linear extension of $P$ starting with $C$.

Lemma 2 Given a poset $P=\left(V,<_{P}\right)$ and a greedy starting chain $C$ of $P$, consider the element $a=\sup _{P}(C)$. If $a \in S_{\max }(P \mid((V-C) \cup\{a\}))$ then $s(P)=s(P \mid(V-C))+1$, that is there exist an optimal linear extension of $P$ starting with $C$.

The previous considerations allow to design a dynamic programming algorithm which recursively generates the states, computes their costs and marks an outgoing arc in $\mathcal{A}$ 
Table 1 Computational results $(n=50 \div 250, p=0.1 \div 0.8)$

\begin{tabular}{|c|c|c|c|c|c|c|c|c|}
\hline$n$ & $p$ & States & $\begin{array}{l}\text { CPPU } \\
\text { Secs. }\end{array}$ & $\frac{U_{B}-L B}{L B}$ & $\frac{U B-x^{*}}{x^{*}}$ & $L B$ & $z^{*}$ & $U B$ \\
\hline \multirow[t]{5}{*}{50} & 0.1 & 168.8 & 4.69 & 0.26 & 0.08 & 15.2 & 17.8 & 19.2 \\
\hline & 0.2 & 705.4 & 11.36 & 0.83 & 0.12 & 10.2 & 16.2 & 18.0 \\
\hline & 0.4 & 62.4 & 2.06 & 0.55 & 0.07 & 9.8 & 13.8 & 14.8 \\
\hline & 0.6 & 10.6 & 0.95 & 0.25 & 0.03 & 9.0 & 10.6 & 11.0 \\
\hline & 0.8 & 1.2 & 0.28 & 0.04 & 0.00 & 6.2 & 6.6 & 6.6 \\
\hline \multirow[t]{5}{*}{100} & 0.1 & 2017.2 & 159.94 & 1.05 & 0.18 & 18.6 & 32.0 & 37.8 \\
\hline & 0.2 & 791.6 & 107.75 & 1.19 & 0.11 & 14.8 & 29.2 & 32.4 \\
\hline & 0.4 & 257.4 & 66.32 & 0.79 & 0.11 & 17.0 & 27.0 & 30.0 \\
\hline & 0.6 & 36.0 & 24.47 & 0.26 & 0.01 & 19.8 & 24.4 & 24.6 \\
\hline & 0.8 & 1.6 & 3.66 & 0.03 & 0.00 & 15.2 & 15.6 & 15.6 \\
\hline \multirow[t]{5}{*}{150} & 0.1 & 4178.2 & 1239.19 & 1.62 & 0.22 & 20.8 & 44.8 & 54.4 \\
\hline & 0.2 & 1339.6 & 633.75 & 2.02 & 0.21 & 17.2 & 41.2 & 49.8 \\
\hline & 0.4 & 338.0 & 233.82 & 0.77 & 0.12 & 25.6 & 40.2 & 45.0 \\
\hline & 0.6 & 80.8 & 169.03 & 0.28 & 0.04 & 29.4 & 36.2 & 37.6 \\
\hline & 0.8 & 6.8 & 32.55 & 0.05 & 0.00 & 20.6 & 21.6 & 21.6 \\
\hline \multirow[t]{5}{*}{200} & 0.1 & 9118.0 & 6300.20 & 2.39 & 0.20 & 19.8 & 55.8 & 67.0 \\
\hline & 0.2 & 1987.8 & 1788.52 & 1.74 & 0.14 & 22.6 & 54.4 & 61.8 \\
\hline & 0.4 & 446.2 & 1478.02 & 0.71 & 0.11 & 35.0 & 53.8 & 59.6 \\
\hline & 0.6 & 104.8 & 747.17 & 0.23 & 0.03 & 40.2 & 47.8 & 49.2 \\
\hline & 0.8 & 15.6 & 154.35 & 0.04 & 0.00 & 27.2 & 28.2 & 28.2 \\
\hline \multirow[t]{5}{*}{250} & 0.1 & 8137.4 & 12031.00 & 2.87 & 0.21 & 21.6 & 68.8 & 83.4 \\
\hline & 0.2 & 2642.0 & 8027.60 & 1.76 & 0.16 & 29.4 & 68.6 & 79.6 \\
\hline & 0.4 & 362.6 & 2992.76 & 0.68 & 0.09 & 42.4 & 64.8 & 70.8 \\
\hline & 0.6 & 204.2 & 4038.94 & 0.27 & 0.03 & 47.2 & 58.4 & 60.2 \\
\hline & 0.8 & 20.4 & 512.64 & 0.04 & 0.00 & 39.0 & 40.4 & 40.4 \\
\hline
\end{tabular}

of the states. The algorithm, looks ahead in the decision process and, in solving the subproblem related to the states $\left(X, C_{i}\right)$, marks the arc $\left(\left(X, C_{i}\right),\left(X^{\prime}, C_{j}\right)\right) \in \mathcal{A}$ for which $f\left(X^{\prime}, C_{j}\right)$ is minimum. Finally, when every state $\left(V, C_{u}\right)$ is solved, the optimal solution can be obtained going along the path on $\mathcal{G}$, starting from the minimum cost state $\left(V, C_{u}^{*}\right)$ and following the marked arcs of $\mathcal{G}$, up to a state without outgoing arcs.

An upper bound $U B\left(X, C_{i}\right)$, issued by the heuristic algorithm, and a lower bound $L B\left(X, C_{i}\right)$, calculated by means of the the defect of $P \mid\left(X-C_{i}\right)$ (i.e. $L B\left(P \mid\left(X-C_{i}\right)\right)=$ de $\left.f_{K}\left(P \mid\left(X-C_{i}\right)\right)-1\right)$ (Gierz and Poguntke, 1983), of the jump number of a minimum jump linear extension of $P \mid X$ starting with $C_{i}$, are used in order to reduce the number of generated states. For the proofs of the theorems and a complete description of the exact algorithm see (Bianco, Dell'Olmo and Giordani, 1994b).

\section{COMPUTATIONAL RESULTS}

The heuristic and exact algorithms have been implemented in $C$ programming language and run on a IBM RISC System/6000. They have been experimentally evaluated on a set of randomly generated test problems, for which, the set of precedence relations, defining a poset $P$, are generated as the transitive closure of a randomly generated direct acyclic graph (dag) without loops, in which it is possible to choose the number $n$ of vertices and the probability threshold $p$ of presence of an arc. That is, for each pair of vertices $(i, j)$ 
with $i<j$ the presence of the arc $(i, j)$ in the dag will be considered if $R N \leq p$, where the $R N$ 's are pseudorandom numbers from a uniform distribution on $[0,1]$.

We have considered test problems of size $n=50,100, \ldots, 250$, and for each size we have randomly generated posets with $p=0.1,0.2,0.4,0.6,0.8$. For each $(n, p)$ combination the results reported are averages over 5 randomly generated problems. The experimental results are summarized in Table 1 , where we have reported the lower bound $L B$, the optimal solution values $z^{*}$ and the heuristic solution values $U B$. We have also measured the heuristic performance as the relative gaps $\left(U B-z^{*}\right) / z^{*},(U B-L B) / L B$, respectively related to the optimal and lower bound values.

Heuristic algorithm often finds optimal solutions: this happens for problems with $p=$ 0.8 , and in general for problem of small size; the worst case is obtained for $n=150$, $p=0.1$ in which the average relative error is $\left(U B-z^{*}\right) / z^{*}=0.22$.

As for the exact algorithm, it arises that problem complexity increases with $n$ and decreases with $p$. Nevertheless, the number of states generated by the algorithm are also strictly related to the relative difference $(U B-L B) / L B$. The worst case occurs for posets for $n=250, p=0.1$ for which the average gap value $(U B-L B) / L B$ is 2.87 and the average number of generated states is 8137.4 ; this case is also the worst one in terms of CPU time for which the time spent is about 12000 seconds, while the worst case in terms of number states occurs for $n=200, p=0.1$ with 9118 generated states.

\section{REFERENCES}

Bianco, L., Dell'Olmo, P. and Giordani, S. (1994a), Exact and Heuristic Algorithms for the Jump Number Problem, in Operations Research Proceedings 1994 (ed. U. Derigs), Springer Verlag, 145-150; Tech. Rep. IASI R.376.

Bianco, L., Dell'Olmo, P. and Giordani, S. (1994b), The Traveling Salesman Problem with Precedence Constraints and Binary Costs. Tech. Rep. IASI R.397.

Bianco, L., Mingozzi, A. and Ricciardelli, S. (1994), Dynamic Programming Strategies for the Traveling Salesman Problem with Time Windows and Precedence Constraints. Opns. Res., to appear.

Faigle, U. and Schrader, R (1985), A setup heuristic for interval orders. Oper. Res. Letters, 4, 185-188.

Gierz, G. and Poguntke, W. (1983), Minimizing Setups for Ordered Sets: a Linear Algebraic Approach. SIAM J. Alg. Disc. Meth., 4, 132-144.

Mitas, J. (1991), Tackling the Jump Number Problem for Interval Orders. Order, 8, 115-132.

Möhring, R.H. (1985), Algorithmic Aspects of Comparability Graphs and Interval Graphs, in Graphs and Order (ed. I. Rival), NATO ASI Series C, vol. 147, D. Reidel Publisher Company, 41-101.

Psaraftis, H. (1980), A dynamic programming solution to the single vehicle many-tomany immediate request dial-a-ride problem. Trans. Sci., 14, 130-154.

Psaraftis, H. (1983), k-Interchange procedures for local search in a precedence-constrained routing problem. E.J.O.R., 13, 391-402.

Pulleyblank, W.R. (1981), Preliminary manuscript.

Savelsberg, M.W.P. (1990), An efficient implementation of local search algorithms for constrained routing problems. E.J.O.R., 47, 75-85. 